\title{
A model for the energetic emission from pulsars
}

\author{
Yu.E. Lyubarskii \\ Institute of Radio Astronomy, Krasnoznamyonnaya St.4, 310002, \\ Kharkov, Ukraine
}

\begin{abstract}
A current sheet separates, beyond the closed part of the pulsar magnetosphere, two half-spaces with oppositely directed magnetic fields. It is shown that reconnection in this sheet may provide a source for high-energy emission.
\end{abstract}

\begin{abstract}
Almost all pulsars are observed only in the radio band but there are exceptions among short-period pulsars. The best known ones are the Crab and Vela pulsars, which are observed, apart from the radio band, in a wide frequency range from optics to gamma rays. Several additional pulsars were found to be gamma sources and also the famous gamma source Geminga was found to be a pulsar. An important point is that these pulsars typically exhibit unusual light curve in the high-energy band; namely two pulses are typically observed during the period. Only few among hundreds of radiopulsars exhibit such a light curve in the radio band. These data strongly support the idea that the energetic emission is concentrated into a fan beam whereas radio emission is concentrated into a pencil beam. It is also important that radio pulses are typically shifted in phase as compared to gamma pulses (the Crab is the only exception). A fan beam of the energetic emission may be naturally produced in a current sheet that separates, beyond the closed region of pulsar magnetosphere, two half-spaces with oppositely directed magnetic fields. A model for this energetic emission from pulsars is outlined here.

According to the most widely accepted view, huge amount of the electronpositron pairs are generated above the pulsar polar cap. This electron-positron plasma streams along the open magnetic field lines, which extent beyond the light cylinder. This magnetized relativistic wind carries away the most part of the spin-down energy of the neutron star. The open magnetic field lines are wrapped backwards so as to keep the velocity of particles sliding along them less than the speed of light. Beyond the light cylinder the magnetic field is predominantly toroidal being oppositely directed in the two hemispheres. The current sheet separates the hemispheres; inside the light cylinder it separates the closed part of the magnetosphere from the open one. The oppositely directed magnetic fields must reconnect through the equatorial current sheet. The reconnection begins at the internal edge of the sheet where it comes to the closed part of the magnetosphere. This occurs at a distance from the star of the order of the light cylinder radius. Due to reconnection the current sheet becomes an active region where magnetic energy releases and supplies energetic emission in the form of a fan beam.
\end{abstract}


The current sheet is supported against the magnetic pressure by the thermal pressure of the ultrarelativistic electron-positron plasma. In rapidly spinning pulsars the magnetic field strength is large at the light cylinder distances therefore the synchrotron cooling rate is high for particles in the sheet. The necessary pressure is provided by a continuous energy release due to a reconnection process. The reconnection is driven by the tearing instability, which growth rate rapidly increases with the decrease of the sheet width. The structure of the sheet is determined by a balance of the heating due to reconnection and the synchrotron cooling.

The reconnection process is conveniently considered in the frame of reference that moves along the current sheet together with the plasma. In this frame reconnection only causes the plasma to subside slowly towards the sheet. The growth rate of the tearing instability in the relativistic plasma is (Zeleny \& Krasnoselskikh 1979)

$$
\gamma \approx \frac{c r_{\mathrm{L}}^{3 / 2}}{a^{5 / 2}},
$$

where $a$ is the sheet width, $r_{\mathrm{L}}=T / e B$ the Larmor radius, $T$ the characteristic energy of the particles. The velocity of the reconnection induced plasma subsidence toward the sheet may be presented then as $u \approx \gamma a=c\left(r_{\mathrm{L}} / a\right)^{3 / 2}$. The subsidence with the velocity $u$ brings into the sheet new plasma and magnetic energy. This energy dissipates inside the sheet and heats the particles. For rapidly spinning pulsars energy release is balanced by synch rotron cooling. Considering energy and particle balance inside the sheet and taking into account that the sheet is in the pressure equilibrium with the surrounding magnetic field, one can obtain a simple system of equation. Solution of this system of equation yields plasma parameters in the current sheet (Lyubarskii 1996).

With the obtained parameters the synchrotron radiation from the sheet may be evaluated easily. The ratio of the Larmor radius to the sheet width turns out to be not too small, $r_{L} / a \sim 0.1$. Therefore the roconnection rate is rather high and energy release is of the order of few percents from the total spin-down power. The primary sheet emission is in the gamma-ray band. Within this powerful fan gamma beam electron-positron pairs are produced due to $\gamma-\gamma$ interaction. These low-energy pairs emit in the infrared and optical bands. Growth of the plasma number density around the sheet due to pair production yields an increase of the particle flux into the sheet and, as a result, a decrease of the particle energy inside the sheet. Then the energy of emitted photons decreases and, provided the number of the produced pairs is large enough, the sheet generates $\mathrm{X}$-rays. Luminosities in the gamma-ray, X-ray and IR-optical bands are estimated and shown to be consistent with those observed from rapidly-spinning pulsars. The fan beam produces a double-peaked pulses and it is very important that observed energetic emission typically exhibits double-peaked pulses.

\section{References}

Lyubarskii, Yu.E. 1996, A\&A, 311, 172

Zeleny L.M., Krasnoselskikh V.V., 1979, Astron.Zh. 56, 819. 